\title{
The Cauchy-Stieltjes integrals in the theory of analytic functions
}

\author{
VLADIMIR I. RYAZANOV
}

(Presented by V. O. Derkach)

\begin{abstract}
We study various Stieltjes integrals as Poisson-Stieltjes, conjugate Poisson-Stieltjes, Schwartz-Stieltjes and Cauchy-Stieltjes and prove theorems on the existence of their finite angular limits a.e. in terms of the Hilbert-Stieltjes integral. These results hold for arbitrary bounded integrands that are differentiable a.e. and, in particular, for integrands of the class $\mathcal{C B V}$ (countably bounded variation).
\end{abstract}

2010 MSC. 30C62, 31A05, 31A20, 31A25, 31B25, 35Q15; Secondary 30E25, 31C05, 34M50, 35F 45 .

Key words and phrases. Stieltjes, Poisson-Stieltjes, Schwartz-Stieltjes, Cauchy-Stieltjes and Hilbert-Stieltjes integrals, harmonic and analytic functions, angular limits.

\section{Introduction}

Recall that a path in $\mathbb{D}:=\{z \in \mathbb{C}:|z|<1\}$ terminating at $\zeta \in \partial \mathbb{D}$ is called nontangential if its part in a neighborhood of $\zeta$ lies inside of an angle in $\mathbb{D}$ with the vertex at $\zeta$. Hence the limit along all nontangential paths at $\zeta \in \partial \mathbb{D}$ also named angular at the point. The latter is a traditional tool of the geometric function theory, see e.g. monographs $[3,11,14,18]$ and $[21]$.

It was proved in the previous paper [24] that a harmonic function $u$ given in the unit disk $\mathbb{D}$ of the complex plane $\mathbb{C}$ has angular limits at a.e. point $\zeta \in \partial \mathbb{D}$ if and only if its conjugate harmonic function $v$ in $\mathbb{D}$ is so. This is the key fact together with Lemma 2 in Section 5 further to establish the existence of the so-called Hilbert-Stieltjes integral for a.e. $\zeta \in \partial \mathbb{D}$ and the corresponding result on the angular limits of Cauchy-Stieltjes integral under fairly general assumptions on integrands, cf. e.g. $[8,19]$ and $[27]$, see also $[4,10,11]$ and [21].

Received 08.08.2017 


\section{Expansion of the Riemann-Stieltjes integral}

First of all, recall a classical definition of the Riemann-Stieltjes integral. Namely, let $\mathrm{I}=[\mathrm{a}, \mathrm{b}]$ be a compact interval in $\mathbb{R}$. A partition $\mathrm{P}$ of $\mathrm{I}$ is a collection of points $t_{0}, t_{1}, \ldots, t_{p} \in \mathrm{I}$ such that $a=t_{0} \leq t_{1} \leq \ldots \leq$ $t_{p}=b$. Now, let $g: \mathrm{I} \rightarrow \mathbb{R}$ and $f: \mathrm{I} \rightarrow \mathbb{R}$ be bounded functions and let $f$ be in addition nondecreasing. The Riemann-Stieltjes integral of $g$ with respect to $f$ is a real number $A$, written $A=\int_{\mathrm{I}} g d f$, if, for every $\varepsilon>0$, there is $\delta>0$ such that

$$
\left|\sum_{k=1}^{p} g\left(\tau_{k}\right)\left[f\left(t_{k}\right)-f\left(t_{k-1}\right)\right]-A\right|<\varepsilon
$$

for every partition $\mathrm{P}=\left\{\mathrm{t}_{0}, \mathrm{t}_{1}, \ldots, \mathrm{t}_{\mathrm{p}}\right\}$ of I with $\left|t_{k}-t_{k-1}\right| \leq \delta, k=$ $1, \ldots, p$, and every collection $\tau_{k} \in\left[t_{k-1}, t_{k}\right], k=1, \ldots, p$. In other words and notations,

$$
\int_{a}^{b} g d f:=\lim _{\delta \rightarrow 0} \sum_{k=1}^{p} g\left(\tau_{k}\right) \cdot \Delta_{k} f \quad \text { as } \quad \delta:=\max _{k=1, \ldots, p}\left|t_{k}-t_{k-1}\right| \rightarrow 0
$$

where $\Delta_{k} f:=f\left(t_{k}\right)-f\left(t_{k-1}\right), k=1, \ldots, p$, if a finite limit in (2.2) exists and it is uniform with respect to partitions $\left\{t_{k}\right\}$ and intermediate points $\left\{\tau_{k}\right\}$. We extend the definition of the Riemann-Stieltjes integral to arbitrary functions $g$ and $f$ for which the limit $(2.2)$ exists. Let us start from the following general fact, cf. e.g. $[1,9,17]$ and $[26]$.

Lemma 1. Let $\mathrm{I}=[\mathrm{a}, \mathrm{b}], g: \mathrm{I} \rightarrow \mathbb{R}$ and $f: \mathrm{I} \rightarrow \mathbb{R}$ be arbitrary functions. If one of the integrals $\int g d f$ and $\int f d g$ exists, then the second one is so and

$$
\int_{\mathrm{I}} g d f+\int_{\mathrm{I}} f d g=g(b) \cdot f(b)-g(a) \cdot f(a) .
$$

Proof. For definiteness, let us assume that there exists the integral $\int f d g$. Then the identity for the arbitrary integral sum of the integral $\int g d f$ :

$$
\begin{aligned}
\sum_{k=1}^{p} g\left(\tau_{k}\right) \cdot\left(f\left(t_{k}\right)-f\left(t_{k-1}\right)\right) & =-g\left(t_{0}\right) f\left(t_{0}\right) \\
& -\sum_{k=1}^{p+1} f\left(t_{k}\right) \cdot\left(g\left(\tau_{k}\right)-g\left(\tau_{k-1}\right)\right)+g\left(t_{p}\right) f\left(t_{p}\right),
\end{aligned}
$$

where $\tau_{0}:=t_{0}$ and $\tau_{p+1}=t_{p}$, implies the desired conclusions. 
By Theorem 13.1.b in [9] we have the significant consequence of Lemma 1.

Proposition 1. Let $\mathrm{I}=[\mathrm{a}, \mathrm{b}]$, a function $f: \mathrm{I} \rightarrow \mathbb{R}$ be absolutely continuous and let $g: \mathrm{I} \rightarrow \mathbb{R}$ be a bounded function whose set of points of discontinuity is of measure zero. Then both integrals $\int_{\mathrm{I}} g d f$ and $\int_{\mathrm{I}} f d g$ exist and the relation (2.3) holds.

Remark 2. It is clear that this formula (2.3) is also valid for complex valued functions on rectifiable curves because their real and imaginary parts as functions of the natural parameter can be considered as real valued functions on segments of $\mathbb{R}$. Moreover, the corresponding statements hold on closed rectfiable Jordan curves $\mathrm{J}$ with the relation

$$
\int_{\mathrm{J}} g d f=-\int_{\mathrm{J}} f d g
$$

where we should apply cyclic partitions $\mathrm{P}$ of $\mathrm{J}$ by collections of cyclic ordered points $\zeta_{0}, \zeta_{1}, \ldots, \zeta_{p}$ on J with $\zeta_{0}=\zeta_{p}$.

\section{On the Poisson-Stieltjes integrals}

Recall that the Poisson kernel is the $2 \pi$-periodic function

$$
P_{r}(\Theta)=\frac{1-r^{2}}{1-2 r \cos \Theta+r^{2}}, r<1, \Theta \in \mathbb{R} .
$$

By Proposition 1 and Remark 1, the Poisson-Stieltjes integral

$$
\mathbb{U}(z)=\mathbb{U}_{\Phi}(z):=\frac{1}{2 \pi} \int_{-\pi}^{\pi} P_{r}(\vartheta-t) d \Phi(t), \quad z=r e^{i \vartheta}, r<1, \vartheta \in \mathbb{R}
$$

is well-defined for $2 \pi$-periodic continuous functions, furthermore, for bounded functions $\Phi: \mathbb{R} \rightarrow \mathbb{R}$ whose set of points of discontinuity is of measure zero because the function $P_{r}(\Theta)$ is continuously differentiable and hence it is absolutely continuous.

Moreover, directly by the definition of the Riemann-Stieltjes integral and the Weierstrass type theorem for harmonic functions, see e.g. Theorem I.3.1 in [7], $U$ is a harmonic function in the unit disk $\mathbb{D}:=\{z \in \mathbb{C}$ : $|z|<1\}$ because the functions $P_{r}(\vartheta-t)$ is the real part of the analytic function

$$
\mathcal{A}_{\zeta}(z):=\frac{\zeta+z}{\zeta-z}, \quad \zeta=e^{i t}, \quad z=r e^{1 \vartheta}, r<1, \vartheta \text { and } t \in \mathbb{R} .
$$


Theorem 1. Let $\Phi: \mathbb{R} \rightarrow \mathbb{R}$ be a $2 \pi$-periodic bounded function whose set of points of discontinuity has measure zero. Suppose that $\Phi$ is differentiable at a point $t_{0} \in \mathbb{R}$. Then

$$
\lim _{z \rightarrow \zeta_{0}} \mathbb{U}_{\Phi}(z)=\Phi^{\prime}\left(t_{0}\right)
$$

along all nontangential paths in $\mathbb{D}$ to the point $\zeta_{0}:=e^{i t_{0}} \in \partial \mathbb{D}$.

Proof. Indeed, by Proposition 1 with $g(t):=\Phi(t)$ and $f(t):=P_{r}(\vartheta-t)$, $t \in \mathbb{R}$, for every fixed $z=r e^{i \vartheta}, r<1, \vartheta \in \mathbb{R}$, we obtain that

$$
\int_{-\pi}^{\pi} P_{r}(\vartheta-t) d \Phi(t)=\int_{-\pi}^{\pi} \Phi(t) \cdot \frac{\partial}{\partial \vartheta} P_{r}(\vartheta-t) d t \quad \forall r \in(0,1), \vartheta \in \mathbb{R}
$$

because of the $2 \pi$-periodicity of the given functions $g$ and $f$ the right hand side in (2.3) is equal to zero, $f \in C^{1}$ and

$$
\frac{\partial}{\partial \vartheta} P_{r}(\vartheta-t)=-\frac{\partial}{\partial t} P_{r}(\vartheta-t) \quad \forall r \in(0,1), \vartheta \text { and } t \in \mathbb{R} .
$$

Now, considering the Poisson integral

$$
u\left(r e^{i \vartheta}\right):=\frac{1}{2 \pi} \int_{-\pi}^{\pi} P_{r}(\vartheta-t) \Phi(t) d t
$$

we see by the Fatou result, see e.g. 3.441 in in [28], p. 53, or Theorem IX.1.2 in [7], that $\frac{\partial}{\partial \vartheta} u(z) \rightarrow \Phi^{\prime}\left(t_{0}\right)$ as $z \rightarrow \zeta_{0}$ along any nontangential path in $\mathbb{D}$ ending at $\zeta_{0}$. Thus, the conclusion follows because just $\mathbb{U}_{\Phi}(z)=$ $\frac{\partial}{\partial \vartheta} u(z)$ by $(3.5)$.

Corollary 1. If $\Phi: \mathbb{R} \rightarrow \mathbb{R}$ is a $2 \pi$-periodic continuous differentiable a.e. function, then (3.4) holds for a.e. $\zeta \in \partial \mathbb{D}$ along all nontangential paths in $\mathbb{D}$ to the point $\zeta$.

Here we denote by $\arg \zeta$ the principal branch of the argument of $\zeta \in \mathbb{C}$ with $|\zeta|=1$, i.e., the unique number $\tau \in(-\pi, \pi]$ such that $\zeta=e^{i \tau}$.

Remark 2. Note that the function of interval $\Phi_{*}([a, b]):=\Phi(b)-$ $\Phi(a)$ generally speaking generates no finite signed measure (charge) if $\Phi$ is not of bounded variation. Hence we cannot apply the known Fatou result on the angular boundary limits directly to the Poisson-Stieltjes integrals, see e.g. Theorem I.D.3 in [11]. 
Corollary 2. If $\Phi: \mathbb{R} \rightarrow \mathbb{R}$ is a $2 \pi$-periodic bounded function that is differentiable a.e., then $\mathbb{U}_{\Phi}(z) \rightarrow \Phi^{\prime}(\arg \zeta)$ as $z \rightarrow \zeta$ for a.e. $\zeta \in \partial \mathbb{D}$ along all nontangential paths in $\mathbb{D}$ to the point $\zeta$.

We call $\Phi: \mathbb{R} \rightarrow \mathbb{C}$ a function of countably bounded variation and write $\Phi \in \mathcal{C B V}(\mathbb{R})$ if there is a countable collection of mutually disjoint intervals $\left(a_{n}, b_{n}\right), n=1,2, \ldots$ on each of which the restriction of $\Phi$ is of bounded variation and the set $\mathbb{R} \backslash \bigcup_{1}^{\infty}\left(a_{n}, b_{n}\right)$ is countable.

Corollary 3. If $\Phi: \mathbb{R} \rightarrow \mathbb{R}$ is a $2 \pi$-periodic bounded function of the class $\mathcal{C B V}(\mathbb{R})$, then $\mathbb{U}_{\Phi}(z) \rightarrow \Phi^{\prime}(\arg \zeta)$ as $z \rightarrow \zeta$ for a.e. $\zeta \in \partial \mathbb{D}$ along all nontangential paths in $\mathbb{D}$ to the point $\zeta$.

\section{On the conjugate Poisson-Stieltjes integrals}

Recall that the conjugate Poisson kernel is the $2 \pi$-periodic function

$$
Q_{r}(\Theta)=\frac{2 r \sin \Theta}{1-2 r \cos \Theta+r^{2}}, r<1, \Theta \in \mathbb{R} .
$$

By Proposition 1 the conjugate Poisson-Stieltjes integral

$$
\mathbb{V}(z)=\mathbb{V}_{\Phi}(z):=\frac{1}{2 \pi} \int_{-\pi}^{\pi} Q_{r}(\vartheta-t) d \Phi(t), \quad z=r e^{i \vartheta}, r<1, \vartheta \in \mathbb{R},
$$

is well-defined for $2 \pi$-periodic bounded functions $\Phi: \mathbb{R} \rightarrow \mathbb{R}$ whose set of points of discontinuity is of measure zero because the function $Q_{r}(\Theta)$ is continuously differentiable and hence it is absolutely continuous. Again, directly by the definition of the Riemann-Stieltjes integral and the Weierstrass type theorem $\mathbb{V}_{\Phi}$ is a conjugate harmonic function for $\mathbb{U}_{\Phi}$ in the unit disk $\mathbb{D}$ because the function $Q_{r}(\vartheta-t)$ is the imaginary part of the same analytic function (3.3).

By Theorem 1 in [24] we have the following significant consequences from Theorem 1 and Corollaries 1-3.

Corollary 4. Let $\Phi: \mathbb{R} \rightarrow \mathbb{R}$ be a $2 \pi$-periodic continuous function that is differentiable a.e. Then $\mathbb{V}_{\Phi}(z)$ has a finite limit $\varphi(\zeta)$ as $z \rightarrow \zeta$ along nontangential paths for a.e. $\zeta \in \partial \mathbb{D}$.

Corollary 5. Let $\Phi: \mathbb{R} \rightarrow \mathbb{R}$ be a $2 \pi$-periodic bounded function that is differentiable a.e. Then $\mathbb{V}_{\Phi}(z)$ has a finite limit $\varphi(\zeta)$ as $z \rightarrow \zeta$ along nontangential paths for a.e. $\zeta \in \partial \mathbb{D}$. 
Corollary 6. Let $\Phi: \mathbb{R} \rightarrow \mathbb{R}$ be a $2 \pi$-periodic bounded function of the class $\mathcal{C B V}(\mathbb{R})$. Then $\mathbb{V}_{\Phi}(z)$ has a finite angular limit $\varphi(\zeta)$ as $z \rightarrow \zeta$ for a.e. $\zeta \in \partial \mathbb{D}$.

The function $\varphi(\zeta)$ will be calculated in the explicit form through $\Phi(\zeta)$ in terms of the so-called Hilbert-Stieltjes integral. To prove this fact we need first to establish one auxiliary result in the next section.

\section{On the Hilbert-Stieltjes integral}

Lemma 2. Let $\Phi: \mathbb{R} \rightarrow \mathbb{R}$ be a $2 \pi$-periodic bounded function whose set of points of discontinuity has measure zero. Suppose that $\Phi$ is differentiable at a point $t_{0} \in \mathbb{R}$. Then the difference

$$
\mathbb{V}_{\Phi}(z)-\frac{1}{\pi} \int_{1-|z|}^{\pi} \frac{d\left\{\Phi\left(t_{0}-t\right)-\Phi\left(t_{0}+t\right)\right\}}{2 \tan \frac{t}{2}}
$$

converges to zero as $z \rightarrow \zeta_{0}:=e^{i t_{0}} \in \partial \mathbb{D}$ along the radius in $\mathbb{D}$ to the point $\zeta_{0}$.

Proof. First of all, in the case of need applying simultaneous rotations $\zeta_{0}$ to $\zeta=e^{i t} \in \partial \mathbb{D}$ and $z \in \mathbb{D}$ in (3.3), we may assume that $t_{0}=0$. Moreover, with no loss of generality we may assume that $\Phi(0)=0$ and $\Phi^{\prime}(0)=0$ because, for the linear function $\Phi_{*}(t):=\Phi(0)+\Phi^{\prime}(0) \cdot t:(-\pi, \pi] \rightarrow \mathbb{R}$ extended $2 \pi$-periodically to $\mathbb{R}, d \Phi_{*}(t) \equiv \Phi^{\prime}(0) d t$, gives identical zero in the difference (5.1) in view of the oddness of the kernel $Q_{r}$ and $\tan \frac{t}{2}$.

Note that by the oddness of $Q_{r}$ we have also that

$\mathbb{V}_{\Phi}(r)=\frac{1}{2 \pi} \int_{-\pi}^{\pi} Q_{r}(-t) d \Phi(t)=\frac{1}{2 \pi} \int_{-\pi}^{\pi} Q_{r}(t) d \Phi(-t), \quad \forall r \in(0,1)$.

Then the difference (5.1) is split into two parts with $\varepsilon=\varepsilon(r):=1-r$ :

$$
\begin{gathered}
\text { I }:=\frac{1}{2 \pi} \int_{-\varepsilon}^{\varepsilon} Q_{r}(t) d \Phi(-t), \\
\mathrm{II}:=\frac{1}{2 \pi} \int_{\varepsilon \leq|t| \leq \pi}\left\{Q_{r}(t)-Q_{1}(t)\right\} d\{\Phi(-t)-\Phi(t)\} .
\end{gathered}
$$


Integrating I by parts, we have by Proposition 1 and Remark 1 and the oddness of $Q_{r}(t)$

$$
\mathrm{I}=\frac{1}{2 \pi} Q_{r}(\varepsilon)\{\Phi(-\varepsilon)-\Phi(\varepsilon)\}+\frac{1}{\pi} \int_{0}^{\varepsilon}\{\Phi(-t)-\Phi(t)\} d Q_{r}(t)
$$

The first summand converges to zero as $\varepsilon \rightarrow 0$ because $|\Phi( \pm \varepsilon)|=o(\varepsilon)$ and

$$
Q_{r}(\varepsilon)=\frac{2 r \sin \varepsilon}{1-2 r \cos \varepsilon+r^{2}}=\frac{2 r \sin \varepsilon}{\varepsilon^{2}+4 r \sin ^{2} \frac{\varepsilon}{2}} \leq 2 \frac{\sin \varepsilon}{\varepsilon^{2}} \leq \frac{2}{\varepsilon} .
$$

To estimate the second summand in (5.2) note that $\sin ^{2} \frac{t}{2} \leq\left[\frac{1-r}{2}\right]^{2}$ and, thus,

$$
\begin{gathered}
Q_{r}^{\prime}(t)=\frac{2 r \cos t}{1-2 r \cos t+r^{2}}-\frac{4 r^{2} \sin ^{2} t}{\left(1-2 r \cos t+r^{2}\right)^{2}} \\
=2 r \frac{\left(1+r^{2}\right) \cos t-2 r}{\left(1-2 r \cos t+r^{2}\right)^{2}}=2 r \frac{(1-r)^{2}-2\left(1+r^{2}\right) \sin ^{2} \frac{t}{2}}{\left(1-2 r \cos t+r^{2}\right)^{2}} \\
\geq 2 r \frac{(1-r)^{2}\left[1-\left(1+r^{2}\right) / 2\right]}{\left(1-2 r \cos t+r^{2}\right)^{2}}=\frac{r(1+r)(1-r)^{3}}{\left(1-2 r \cos t+r^{2}\right)^{2}},
\end{gathered}
$$

i.e., $Q_{r}^{\prime}(t)>0$ for all $t \in[0, \varepsilon]$. Since $Q_{r}(t)$ is smooth, it is strictly increasing on $[0, \varepsilon]$. Hence the modulus of the second summand has the upper bound

$$
\frac{1}{\pi} \cdot Q_{r}(\varepsilon) \cdot \sup _{t \in[0, \varepsilon]}\{|\Phi(-t)|+|\Phi(t)|\} \leq \frac{1}{\pi} \cdot \frac{2}{\varepsilon} \cdot o(\varepsilon)=o(1)
$$

where the inequality follows by (5.3). Thus, the second summand in (5.2) also converges to zero as $\varepsilon \rightarrow 0$.

Now, by oddness of the kernels $Q_{r}(t), r \in(0,1)$ and $Q_{1}(t)$ we obtain that

$$
\mathrm{II}:=\frac{1}{\pi} \int_{\varepsilon}^{\pi}\left\{Q_{r}(t)-Q_{1}(t)\right\} d\{\Phi(-t)-\Phi(t)\}
$$

where

$$
\begin{aligned}
& Q_{1}(t)-Q_{r}(t)=\frac{2 \sin t}{2(1-\cos t)}-\frac{2 r \sin t}{\varepsilon^{2}+2 r(1-\cos t)} \\
= & \frac{2 \sin t}{4 \sin ^{2} \frac{t}{2}}-\frac{2 r \sin t}{\varepsilon^{2}+4 r \sin ^{2} \frac{t}{2}}=\frac{2 \varepsilon^{2} \sin t}{4\left(\varepsilon^{2}+4 r \sin ^{2} \frac{t}{2}\right) \sin ^{2} \frac{t}{2}} .
\end{aligned}
$$


Integrating by parts, we see that the latter integral is equal to $\left\{Q_{1}(\varepsilon)-Q_{r}(\varepsilon)\right\} \cdot\{\Phi(-\varepsilon)-\Phi(\varepsilon)\}+\int_{\varepsilon}^{\pi}\{\Phi(-t)-\Phi(t)\} d\left\{Q_{1}(t)-Q_{r}(t)\right\}$.

Here the first summand converges to zero because $\Phi(-\varepsilon)-\Phi(\varepsilon)=o(\varepsilon)$ and

$$
Q_{1}(\varepsilon)-Q_{r}(\varepsilon)=\frac{2 \varepsilon^{2} \sin \varepsilon}{4\left(\varepsilon^{2}+4 r \sin ^{2} \frac{\varepsilon}{2}\right) \sin ^{2} \frac{\varepsilon}{2}} \sim \frac{1}{\varepsilon} \quad \text { as } \quad \varepsilon \rightarrow 0 .
$$

Thus, it remains to estimate the integral

$$
\text { III }:=\int_{\varepsilon}^{\pi}\{\Phi(-t)-\Phi(t)\} d\left\{Q_{1}(t)-Q_{r}(t)\right\}=\int_{\varepsilon}^{\pi} \varphi(t) d \alpha_{r}(t)
$$

where $\varphi(t)=\Phi(-t)-\Phi(t)$ and $\alpha_{r}(t)=Q_{1}(t)-Q_{r}(t)$. To make it first of all note that $\alpha_{r}^{\prime}(t)<0$ for $t \in(\varepsilon, \pi)$ because of

$$
\begin{gathered}
\alpha_{r}^{\prime}(t)=\frac{2 \varepsilon^{2} \cos t}{4\left(\varepsilon^{2}+4 r \sin ^{2} \frac{t}{2}\right) \sin ^{2} \frac{t}{2}}-\frac{2 \varepsilon^{2} \sin ^{2} t\left(\varepsilon^{2}+8 r \sin ^{2} \frac{t}{2}\right)}{4\left[\left(\varepsilon^{2}+4 r \sin ^{2} \frac{t}{2}\right) \sin ^{2} \frac{t}{2}\right]^{2}} \\
=2 \cdot \frac{\varepsilon^{2}}{\delta^{2}} \cdot\left[\cos t \cdot \sin ^{2} \frac{t}{2} \cdot\left(\varepsilon^{2}+4 r \sin ^{2} \frac{t}{2}\right)-\sin ^{2} t \cdot\left(\varepsilon^{2}+8 r \sin ^{2} \frac{t}{2}\right)\right] \\
=2 \cdot \frac{\varepsilon^{2}}{\delta^{2}} \cdot\left[\varepsilon^{2} \cdot\left(\cos t \cdot \sin ^{2} \frac{t}{2}-\sin ^{2} t\right)-4 r \sin ^{2} \frac{t}{2} \cdot\left(2 \sin ^{2} t-\cos t \cdot \sin ^{2} \frac{t}{2}\right)\right] \\
=2 \cdot \frac{\varepsilon^{2}}{\delta^{2}} \cdot\left[\varepsilon^{2} \cdot \sin ^{2} \frac{t}{2} \cdot\left(\cos t-4 \cos ^{2} \frac{t}{2}\right)-4 r \sin ^{4} \frac{t}{2} \cdot\left(8 \cos ^{2} \frac{t}{2}-\cos t\right)\right] \\
=-2 \cdot \frac{\varepsilon^{2}}{\delta^{2}} \cdot \sin ^{2} \frac{t}{2} \cdot\left[\varepsilon^{2} \cdot\left(1+2 \cos ^{2} \frac{t}{2}\right)+4 r \sin ^{2} \frac{t}{2} \cdot\left(1+6 \cos ^{2} \frac{t}{2}\right)\right]
\end{gathered}
$$

where we many times applied the trigonometric identities $\sin t=2 \sin \frac{t}{2} \cos \frac{t}{2}$ and $1-\cos t=2 \sin ^{2} \frac{t}{2}, 1+\cos t=2 \cos ^{2} \frac{t}{2}$, and the notation

$$
\delta:=2 \sin ^{2} \frac{t}{2}\left(\varepsilon^{2}+4 r \sin ^{2} \frac{t}{2}\right) .
$$

The above expression for $\alpha_{r}^{\prime}(t)$ also implies that $\left|\alpha_{r}^{\prime}(t)\right| \leq c \cdot \frac{\varepsilon^{2}}{t^{4}}$. Thus,

$$
|\mathrm{III}| \leq c \cdot \varepsilon^{2} \int_{\varepsilon}^{\pi}|\varphi(t)| \frac{d t}{t^{4}}
$$


Let us fix an arbitrary $\epsilon>0$ and choose a small enough $\eta>0$ such that $|\varphi(t)| / t<\epsilon$ for all $t \in(0, \eta)$. Note that we may assume here that $\varepsilon<\eta^{2} \sqrt{ } \epsilon$ for small enough $\varepsilon$. Consequently, we have the following estimates

$$
|\mathrm{III}| \leq c \cdot \varepsilon^{2} \cdot \epsilon \int_{\varepsilon}^{\eta} \frac{d t}{t^{3}}+c \cdot \varepsilon^{2} \int_{\eta}^{\pi}|\varphi(t)| \frac{d t}{t^{4}} \leq \frac{c}{2} \cdot \epsilon+c \pi \cdot \epsilon \cdot M
$$

where $M=\sup _{t \in[0, \pi]}|\varphi(t)|$. In view of arbitrariness of $\varepsilon$ and $\epsilon$, we conclude that the integral III converges to zero as $\varepsilon \rightarrow 0$.

Theorem 2. Let $\Phi: \mathbb{R} \rightarrow \mathbb{R}$ be a $2 \pi$-periodic bounded function. Suppose that $\Phi$ is differentiable a.e. Then

$$
\lim _{z \rightarrow \xi} \mathbb{V}_{\Phi}(z)=\frac{1}{\pi} \int_{0}^{\pi} \frac{d\{\Phi(\tau-t)-\Phi(\tau+t)\}}{2 \tan \frac{t}{2}}, \quad \xi:=e^{i \tau} \in \partial \mathbb{D},
$$

for a.e. $\tau \in \mathbb{R}$ along all nontangential paths in $\mathbb{D}$ to the point $\xi$.

Here the singular integral from the right hand side in (5.5) is understood as a limit of the corresponding proper integrals (principal value by Cauchy):

$$
\mathbb{H}_{\Phi}(\tau):=\frac{1}{\pi} \lim _{\varepsilon \rightarrow+0} \int_{\varepsilon}^{\pi} \frac{d\{\Phi(\tau-t)-\Phi(\tau+t)\}}{2 \tan \frac{t}{2}}=\frac{1}{2 \pi} \lim _{\varepsilon \rightarrow 0} \int_{|\tau-t| \geq \varepsilon} \frac{d \Phi(t)}{\tan \frac{\tau-t}{2}} .
$$

We call it as the Hilbert-Stieltjes integral of the function $\Phi$ at the point $\tau$.

Proof. The conclusion of Theorem 2 follows immediately from Lemma 2 and Corollary 5.

Corollary 7. The Hilbert-Stieltjes integral converges a.e. for every $2 \pi$-periodic bounded function $\Phi: \mathbb{R} \rightarrow \mathbb{R}$ that is differentiable a.e.

Remark 3. In particular, the conclusions of Theorem 2 as well as Corollary 7 hold for every $2 \pi$-periodic bounded function of the class $\mathcal{C B} \mathcal{V}(\mathbb{R})$.

Of course, Lemmas 1-2, Theorems 1-2, Corollaries 1-7 and the definition of the Hilbert-Stieltjes integral are extended in the natural way to complex valued functions $\Phi$. 


\section{On Schwartz-Stieltjes and Cauchy-Stieltjes integrals}

Given a $2 \pi$-periodic bounded function $\Phi: \mathbb{R} \rightarrow \mathbb{R}$ whose set of points of discontinuity has measure zero, the Schwartz-Stieltjes integral

$$
\mathbb{S}_{\Phi}(z):=\frac{1}{2 \pi} \int_{-\pi}^{\pi} \frac{e^{i t}+z}{e^{i t}-z} d \Phi(t), \quad z \in \mathbb{D}
$$

is well-defined by the previous sections and the function $\mathbb{S}_{\Phi}(z)$ is analytic by the definition of the Riemann-Stieltjes integral and the Weierstrass theorem, see e.g. Theorem I.1.1 in [7]. By Theorem 2 and Corollary 2 we have also the following.

Corollary 8. Let $\Phi: \mathbb{R} \rightarrow \mathbb{R}$ be a $2 \pi$-periodic bounded function that is differentiable a.e. Then $\mathbb{S}_{\Phi}(z)$ has finite angular limit $\Phi^{\prime}(\arg \zeta)+i$. $\mathbb{H}_{\Phi}(\arg \zeta)$ as $z \rightarrow \zeta$ for a.e. $\zeta \in \partial \mathbb{D}$.

It is clear that the definition (6.1), as well as Corollary 8 , is extended in the natural way to the case of the complex valued functions $\Phi$.

Given a $2 \pi$-periodic bounded function $\Phi: \mathbb{R} \rightarrow \mathbb{C}$ whose set of points of discontinuity has measure zero, we see that the integral

$$
\mathbb{C}_{\Phi}(z):=\frac{1}{2 \pi} \int_{-\pi}^{\pi} \frac{e^{i t} d \Phi(t)}{e^{i t}-z}, \quad z \in \mathbb{D}
$$

is also well-defined and we call it by the Cauchy-Stieltjes integral. It is easy to see that $\mathbb{C}_{\Phi}(z)=\frac{1}{2} \mathbb{S}_{\Phi}(z)$.

Corollary 9. Let $\Phi: \mathbb{R} \rightarrow \mathbb{C}$ be a $2 \pi$-periodic bounded function that is differentiable a.e. Then

$$
\lim _{z \rightarrow \zeta} \mathbb{C}_{\Phi}(z)=\frac{1}{2}\left\{\Phi^{\prime}(\arg \zeta)+i \cdot \mathbb{H}_{\Phi}(\arg \zeta)\right\}
$$

for a.e. $\zeta \in \partial \mathbb{D}$ along all nontangential paths in $\mathbb{D}$ to the point $\zeta$.

In this connection, note that the Hilbert-Stieltjes integral can be described in another way for functions $\Phi$ of bounded variation.

Namely, let us denote by $C\left(\zeta_{0}, \varepsilon\right), \varepsilon \in(0,1), \zeta_{0} \in \partial \mathbb{D}$, the rest of the unit circle $\partial \mathbb{D}$ after removing its $\operatorname{arc} A\left(\zeta_{0}, \varepsilon\right):=\left\{\zeta \in \partial \mathbb{D}:\left|\zeta-\zeta_{0}\right|<\varepsilon\right\}$ and, setting

$$
\mathrm{I}_{\Phi}\left(\zeta_{0}, \varepsilon\right)=\frac{1}{2 \pi} \int_{C\left(\zeta_{0}, \varepsilon\right)} \frac{\zeta d \Phi_{*}(\zeta)}{\zeta-\zeta_{0}}, \quad \zeta_{0} \in \partial \mathbb{D}, \quad \text { where } \Phi_{*}(\zeta):=\Phi(\arg \zeta),
$$


define the singular integral of the Cauchy-Stieltjes type

$$
\mathbb{I}_{\Phi}\left(\zeta_{0}\right)=\frac{1}{2 \pi} \int_{\partial \mathbb{D}} \frac{\zeta d \Phi}{\zeta-\zeta_{0}}, \quad \zeta_{0} \in \partial \mathbb{D},
$$

as a limit of the integrals $\mathrm{I}\left(\zeta_{0}, \varepsilon\right)$ as $\varepsilon \rightarrow 0$. By paper [19] we have that

$$
\lim _{z \rightarrow \zeta} \mathbb{C}_{\Phi}(z)=\frac{1}{2} \cdot \Phi^{\prime}(\arg \zeta)+i \cdot \mathbb{I}_{\Phi}(\zeta)
$$

for a.e. $\zeta \in \partial \mathbb{D}$ along all nontangential paths in $\mathbb{D}$ to the point $\zeta$. Comparing the relations (6.3) and (6.4), we come to the following conclusion.

Corollary 10. Let $\Phi: \mathbb{R} \rightarrow \mathbb{C}$ be a $2 \pi$-periodic function with bounded variation on $[-\pi, \pi]$. Then for a.e. $\tau \in[-\pi, \pi]$

$$
\mathbb{H}_{\Phi}(\tau)=2 \cdot \mathbb{I}_{\Phi}\left(e^{i \tau}\right)
$$

\section{Representation of the Luzin construction}

The following deep (non-trivial) result of Luzin was one of the main theorems of his dissertation, see e.g. his paper [12], dissertation [13], p. 35 , and its reprint [14, p. 78], where one may assume that $\Phi(0)=\Phi(1)=$ 0 , cf. also [25].

Theorem A. For any measurable function $\varphi:[0,1] \rightarrow \mathbb{R}$, there is a continuous function $\Phi:[0,1] \rightarrow \mathbb{R}$ such that $\Phi^{\prime}=\varphi$ a.e.

Just on the basis of Theorem A, Luzin proved the next significant result of his dissertation, see e.g. [14, p. 80], that was key to establish the corresponding result on the boundary value Hilbert problem for analytic functions in [22].

Theorem B. Let $\varphi(\vartheta)$ be real, measurable, almost everywhere finite and have the period $2 \pi$. Then there exists a harmonic function $U$ in the unit disk $\mathbb{D}$ such that $U(z) \rightarrow \varphi(\vartheta)$ for a.e. $\vartheta$ as $z \rightarrow e^{i \vartheta}$ along any nontangential path.

Note that the Luzin dissertation was published in Russian as the book [14] with comments of his pupils Bari and Men'shov only after his death. A part of its results was also printed in Italian [15]. However, Theorem A was published with a complete proof in English in the book 
[26] as Theorem VII (2.3). Hence Frederick Gehring in [6] has rediscovered Theorem B and his proof on the basis of Theorem A has in fact coincided with the original proof of Luzin. Since the proof is very short and nice and has a common interest, we give it for completeness here.

Proof. By Theorem A we can find a continuous function $\Phi(\vartheta)$ such that $\Phi^{\prime}(\vartheta)=\varphi(\vartheta)$ for a.e. $\vartheta$. Considering the Poisson integral

$$
u\left(r e^{i \vartheta}\right)=\frac{1}{2 \pi} \int_{0}^{2 \pi} \frac{1-r^{2}}{1-2 r \cos (\vartheta-t)+r^{2}} \Phi(t) d t
$$

for $0<r<1, u(0):=0$, we see by the Fatou result, see e.g. 3.441 in [28], p. 53, or Theorem IX.1.2 in [7], that $\frac{\partial}{\partial \vartheta} u(z) \rightarrow \Phi^{\prime}(\vartheta)$ as $z \rightarrow e^{i \vartheta}$ along any nontangential path whenever $\Phi^{\prime}(\vartheta)$ exists. Thus, the conclusion follows for the function $U(z)=\frac{\partial}{\partial \vartheta} u(z)$.

Remark 4. Note that the given function $U$ is harmonic in the punctured unit disk $\mathbb{D} \backslash\{0\}$ because the function $u$ is harmonic in $\mathbb{D}$ and the differential operator $\frac{\partial}{\partial \vartheta}$ is commutative with the Laplace operator $\Delta$. Setting $U(0)=0$, we see that

$U\left(r e^{i \vartheta}\right)=-\frac{r}{\pi} \int_{0}^{2 \pi} \frac{\left(1-r^{2}\right) \sin (\vartheta-t)}{\left(1-2 r \cos (\vartheta-t)+r^{2}\right)^{2}} \Phi(t) d t \rightarrow 0 \quad$ as $r \rightarrow 0$,

i.e. $U(z) \rightarrow U(0)$ as $z \rightarrow 0$, and, moreover, the integral of $U$ over each circle $|z|=r, 0<r<1$, is equal to zero. Thus, by the criterion for a harmonic function on the averages over circles we have that $U$ is harmonic in $\mathbb{D}$. The alternative argument for the latter is the removability of isolated singularities for harmonic functions, see e.g. [16].

Corollary 5.1 in $[22]$ has strengthened Theorem B as the next, see also [23].

Theorem C. For each (Lebesgue) measurable function $\varphi: \partial \mathbb{D} \rightarrow \mathbb{R}$, the space of all harmonic functions $u: \mathbb{D} \rightarrow \mathbb{R}$ with the angular limits $\varphi(\zeta)$ for a.e. $\zeta \in \partial \mathbb{D}$ has the infinite dimension.

Remark 5. One can find in [25] more refined results which are counterparts of Theorems A, B and C in terms of logarithmic capacity that makes possible to extend the theory of boundary value problems 
to the so-called $A$-harmonic functions corresponding to generalizations of the Laplace equation in inhomogeneous and anisotropic media, see also [29].

By the well-known Lindelöf maximum principle, see e.g. Lemma 1.1 in [5], it follows the uniqueness theorem for the Dirichlet problem in the class of bounded harmonic functions $u$ on the unit disk $\mathbb{D}=\{z \in \mathbb{C}:|z|<1\}$. In general there is no uniqueness theorem in the Dirichlet problem for the Laplace equation even under zero boundary data.

Many such nontrivial solutions for the Laplace equation can be given just by the Poisson-Stieltjes integral

$$
\mathbb{U}_{\Phi}(z):=\frac{1}{2 \pi} \int_{0}^{2 \pi} P_{r}(\vartheta-t) d \Phi(t), \quad z=r e^{i \vartheta}, r<1,
$$

with an arbitrary singular function $\Phi:[0,2 \pi] \rightarrow \mathbb{R}$, i.e., where $\Phi$ is of bounded variation and $\Phi^{\prime}=0$ a.e. Indeed, by the Fatou theorem, see e.g. Theorem I.D.3.1 in [11], $\mathbb{U}_{\Phi}(z) \rightarrow \Phi^{\prime}(\Theta)$ as $z \rightarrow e^{i \Theta}$ along any nontangential path whenever $\Phi^{\prime}(\Theta)$ exists, i.e. $\mathbb{U}_{\Phi}(z) \rightarrow 0$ as $z \rightarrow e^{i \Theta}$ for a.e. $\Theta \in[0,2 \pi]$ along any nontangential paths for every singular function $\Phi$.

Example 1. The first natural example is given by the formula (7.1) with $\Phi(t)=\varphi(t / 2 \pi)$ where $\varphi:[0,1] \rightarrow[0,1]$ is the well-known Cantor function, see e.g. [2] and further references therein.

Example 2. However, the simplest example of such a kind is just

$$
u(z):=P_{r}\left(\vartheta-\vartheta_{0}\right)=\frac{1-r^{2}}{1-2 r \cos \left(\vartheta-\vartheta_{0}\right)+r^{2}}, \quad z=r e^{i \vartheta}, r<1 .
$$

We see that $u(z) \rightarrow 0$ as $z \rightarrow e^{i \Theta}$ for all $\Theta \in(0,2 \pi)$ except $\Theta=\vartheta_{0}$.

The construction of Luzin can be described as the Poisson-Stieltjes integral.

Theorem 3. The harmonic function $U$ in Theorem $B$ has the representation $\mathbb{U}_{\Phi}(z)=\frac{1}{2 \pi} \int_{-\pi}^{\pi} P_{r}(\vartheta-t) d \Phi(t) \quad \forall z=r e^{i \vartheta}, r \in(0,1), \vartheta \in[-\pi, \pi]$, 
where $\Phi:[-\pi, \pi] \rightarrow \mathbb{R}$ is the continuous Luzin function with $\Phi^{\prime}=\varphi$ a.e.

Corollary 11. The conjugate harmonic function $\mathbb{V}_{\Phi}$ has finite angular limits

$$
\lim _{z \rightarrow \zeta} \mathbb{V}_{\Phi}(z)=\mathbb{H}_{\Phi}(\arg \zeta) \quad \text { for a.e. } \quad \zeta \in \partial \mathbb{D}
$$

Proof. Indeed, choosing in Proposition $1 g(t)=\Phi(t)$ and $f(t)=P_{r}(\vartheta-t)$, $t \in[-\pi, \pi]$, for every fixed $z=r e^{i \vartheta}, r<1, \vartheta \in[-\pi, \pi]$, we obtain that

$$
\int_{-\pi}^{\pi} \Phi(t) \cdot \frac{\partial}{\partial \vartheta} P_{r}(\vartheta-t) d t=\int_{-\pi}^{\pi} P_{r}(\vartheta-t) d \Phi(t) \quad \forall r \in(0,1), \vartheta \in[-\pi, \pi]
$$

because by the $2 \pi$-periodicity of the given function $g$ and $f$ the right hand side in (2.3) is equal to zero, $f \in C^{1}$, and

$$
\frac{\partial}{\partial \vartheta} P_{r}(\vartheta-t)=-\frac{\partial}{\partial t} P_{r}(\vartheta-t) \quad \forall r \in(0,1), \vartheta \in[-\pi, \pi], t \in[-\pi, \pi] .
$$

The relation (7.2) follows from (7.3) because it was in the proof of Theorem B

$U(z)=\frac{1}{2 \pi} \int_{-\pi}^{\pi} \Phi(t) \cdot \frac{\partial}{\partial \vartheta} P_{r}(\vartheta-t) d t \quad \forall z=r e^{i \vartheta}, r \in(0,1), \vartheta \in[-\pi, \pi]$.

\section{References}

[1] A. A. Borovkov, Probability theory, Transl. from 1986 Russian original by A. Borovkova and revised by the author, Gordon and Breach Science Publishers, Amsterdam, 1998

[2] O. Dovgoshey, O. Martio, V. Ryazanov, M. Vuorinen, The Cantor function // Expo. Math., 24 (2006), 1-37.

[3] P. L. Duren, Theory of Hp spaces // Pure and Applied Mathematics, 38, Academic Press, New York-London, 1970.

[4] E. M. Dyn'kin, Methods of the theory of singular integrals (the Hilbert transform and Calderron-Zygmund theory) // Current problems in mathematics. Fundamental directions, 15, 197-292, 303, Itogi Nauki i Tekhniki, Akad. Nauk SSSR, Vsesoyuz. Inst. Nauchn. i Tekhn. Inform., Moscow, 1987 [in Russian].

[5] J. B. Garnett, D. E. Marshall, Harmonic Measure, Cambridge Univ. Press, 2005.

[6] F. W. Gehring, On the Dirichlet problem // Michigan Math. J., 3 (1955-1956), 201. 
[7] G. M. Goluzin, Geometric theory of functions of a complex variable, Transl. of Math. Monographs, 26, American Mathematical Society, Providence, R.I., 1969.

[8] V. P. Havin, Boundary properties of integrals of Cauchy type and of conjugate harmonic functions in regions with rectifable boundary // Mat. Sb. (N.S.), 68 (110) (1965), 499-517 [in Russian].

[9] P. L. Henneken, A. Tortra, Teoriya veroyatnostei i nekotorye ee prilozheniya // Probability theory and some of its applications, Nauka, Moscow, 1974 [in Russian]; transl. from Hennequin P.L., Tortrat A., Theorie des probabilites et quelques applications, [in French] Masson et Cie, Editeurs, Paris, 1965.

[10] S. V. Kislyakov, Classical problems of Fourier analysis // Current problems in mathematics. Fundamental directions, 15, 135-195, 303, Itogi Nauki i Tekhniki, Akad. Nauk SSSR, Vsesoyuz. Inst. Nauchn. i Tekhn. Inform., Moscow, 1987 [in Russian].

[11] P. Koosis, Introduction to $H_{p}$ spaces, 2nd ed., Cambridge Tracts in Mathematics, 115, Cambridge Univ. Press, Cambridge, 1998.

[12] N. N. Luzin, On the main theorem of integral calculus // Mat. Sb., 28 (1912), 266-294 [in Russian].

[13] N. N. Luzin, Integral and trigonometric series, Dissertation, Moskwa, 1915 [in Russian].

[14] N. N. Luzin, Integral and trigonometric series, Editing and commentary by N.K. Bari and D.E. Men'shov. Gosudarstv. Izdat. Tehn.-Teor. Lit., MoscowLeningrad, 1951 [in Russian].

[15] N. Luzin, Sur la notion de l'integrale // Annali Mat. Pura e Appl., 26 (1917), No. 3, 77-129.

[16] R. Nevanlinna, Eindeutige analytische Funktionen, Ann Arbor, Michigan, 1944.

[17] W. F. Pfeffer, Integration by parts for the generalized Riemann-Stieltjes integral // J. Austral. Math. Soc. Ser. A, 34 (1983), No. 2, 229-233.

[18] Ch. Pommerenke, Boundary behaviour of conformal maps, Fundamental Principles of Mathematical Sciences, 299, Springer-Verlag, Berlin, 1992.

[19] I. Privaloff, Sur l'integrale du type de Cauchy-Stieltjes // Bull. Acad. Sci. URSS. Ser. Math., 4 (1940), 261-276 [in Russian].

[20] I. I. Priwalow, Introduction in the theory of functions of one complex variable, ed. 12, Nauka, Moscow, 1977 [in Russian].

[21] I. I. Priwalow, Randeigenschaften analytischer Funktionen // Hochschulbücher für Mathematik, 25, Deutscher Verlag der Wissenschaften, Berlin, 1956.

[22] V. Ryazanov, On the Riemann-Hilbert Problem without Index // Ann. Univ. Bucharest, Ser. Math., 5 (LXIII) (2014), No. 1, 169-178.

[23] V. Ryazanov, Infinite dimension of solutions of the Dirichlet problem // Open Math. (the former Central European J. Math.), 13 (2015), No. 1, 348-350.

[24] V. Ryazanov, Correlation of boundary behavior of conjugate harmonic functions // Proc. IAMM NASU, 31 (2017) (to appear); see also arXiv.org: 1710.00323v1 [math.CV].

[25] V. Ryazanov, A. Yefimushkin, On the Riemann-Hilbert Problem for the Beltrami Equations // Contemp. Math., 667 (2016), 299-316.

[26] S. Saks, Theory of the integral, Warsaw, 1937; Dover Publications Inc., New York, 1964. 
[27] V. Smirnoff, Sur les valeurs limites des fonctions, regulieres a l'interieur d'un cercle // J. Soc. Phys.-Math. Leningrade, 2 (1929), No. 2, 22-37.

[28] A. Zygmund, Trigonometric series, Wilno, 1935.

[29] A. Yefimushkin, On Neumann and Poincare problems in A-harmonic analysis // Advances in Analysis, 1 (2016), No. 2, 114-120; see also arXiv.org: 1608.08457v1 [math.CV] 30 Aug 2016, 1-14.

\section{CONTACT INFORMATION}

Vladimir I.

Ryazanov
Institute of Applied Mathematics

and Mechanics of the NAS of Ukraine,

Slavyansk, Ukraine

E-Mail: vl.ryazanov1@gmail.com 